\title{
A variable step size subband affine projection algorithm with dynamic selection of subband filters
}

\author{
Chang Liu ${ }^{\text {a) }}$ Zishu He, Hefa Zhang, Hongshu Liao, and Wei Xia \\ School of Electronic Engineering, University of Electronic Science and Technology \\ of China, no.2006 Xiyuan Road, Chengdu, 611731 P.R.China \\ a)chaneaaa@163.com
}

\begin{abstract}
In this paper, we present a novel variable step size subband affine projection (VSS-SAP) algorithm which dynamically selects subband filters in order to reduce the computational complexity and improve the convergence performance. It is called as dynamic selection variable step size subband affine projection algorithm (DS-VSS-SAP). The optimum selection of subband filters is derived by the largest decrease between the successive mean square deviations (MSDs) at every iteration. Simulation results show that the proposed algorithm has a lower misadjustment compared with the existing SAP algorithms. In addition, it retains a lower overall computational complexity.
\end{abstract}

Keywords: adaptive filters, dynamic selection of subband filters, subband affine projection

Classification: Science and engineering for electronics

\section{References}

[1] H. Choi and H. D. Bae, "Subband affine projection algorithm for acoustic echo cancellation," EURASIP Journal on Advances in Signal Processing, 2007.

[2] H. Choi, S. H. Han, and H. D. Bae, "Subband adaptive filtering with maximal decimation using an affine projection algorithm," IEICE Trans. Commun., vol. E89-B, no. 5, pp. 1477-1485, May 2006.

[3] M. Karthik, A. K. Krishna, K. H. Li, and S. George, "Delay coefficients based variable step size algorithm for subband affine projection adaptive filters," IEICE Electron. Express, vol. 6, no. 1, pp. 20-26, Jan. 2009.

[4] M. Karthik, K. H. Li, and S. George, "Delay coefficients based variable regularization subband affine projection algorithm in acoustic echo cancellation application," IEICE Trans. Fundamentals, vol. E92-A, no. 7, pp. 1699-1703, July 2009.

[5] S. E. Kim, Y. S. Choi, M. K. Song, and W. J. Song, "A subband adaptive filtering algorithm employing dynamic selection of subband filters," IEEE Signal process. Lett., vol. 17, no. 3, pp. 245-248, March 2010.

[6] S. J. Kong, K. Y. Hwang, and W. J. Song, "An affine projection algorithm with dynamic selection of input vectors," IEEE Signal process. Lett., vol. 14, no. 8, pp. 529-532, Aug. 2007. 
[7] J. Ni and F. Li, "A variable step-size matrix normalized subband adaptive filter," IEEE Trans. Speech Audio Process., vol. 18, no. 6, pp. 1290-1299, Aug. 2010.

\section{Introduction}

The affine projection (AP) algorithm provides an improved convergence rate over the least mean square (LMS) algorithm and has less computational complexity than the recursive least square (RLS) algorithm. Subband adaptive filtering (SAF) is typically employed as a method to overcome the problems of high complexity and slow convergence for long adaptive filters (AF). Recently, a subband AP (SAP) algorithm has been reported in [1, 2] by combining the characteristics of the AP and SAF. Furthermore, it makes use of the polyphase decomposition of the AF and noble identities to avoid the use of cross filters. A delay coefficients-based variable step size subband AP algorithm (DC-VSS-SAP) [3] which is based on the use of the variable step size and delay coefficients, prevents the AF from diverging in acoustic echo cancellation (AEC) applications. In addition, a new VSS-SAP algorithm which is called delay coefficients based variable regularization SAP (DC-VR-SAP) is published in [4]. It inherits the advantages of both the methods of delay coefficients (DC) and variable regularization (VR) for AEC application.

The computational complexity is a weak point in the implementations of SAP. Both the high projection order of SAP and extremely long unknown system such as AEC application result in huge complexity. In order to reduce computational complexity, normalized subband adaptive filter algorithm employing dynamic selection of subband filters (DS- NSAF) has been proposed in [5]. In this paper, we present a novel VSS-SAP algorithm whose main goal is to reduce the computational complexity by selecting a significant subset of the subband filters. We call this proposed VSS-SAP as dynamic selection VSS-SAP (DS-VSS-SAP). It dynamically selects the significant subband filters so as to maximize the decrease between the successive mean square deviations (MSDs) at every iteration. Furthermore, its variable step size is obtained by the method of delay coefficients [3]. Simulation results show that it has a lower misadjustment compared with the existing SAP algorithms.

The paper is organized as follows. Section 2 introduces the SAP [1, 2] and DC-VSS-SAP [3] algorithms. Section 3 presents the proposed DS-VSS-SAP algorithm and computer simulations are illustrated in Section 4. Conclusions are given in Section 5.

\section{SAP and DC-VSS-SAP algorithms}

The system model of SAP is illustrated in Fig. 3 of [2] which uses the polyphase decomposition of the AF and noble identities. The notation in this paper will be consistent with the ones used in [2] and [3]. The system contains $M$ subbands and is used to estimate the unknown system de- 
noted by an $N \times 1$ vector $\mathbf{s}^{*}$. The projection order of SAP is $P$ and subband projection order is denoted by $P_{s}=P / M$. Assuming that the indices $i, j \in[0, \cdots, M-1]$ through out this paper. $M$ polyphase components of $\mathbf{s}^{*}$ are denoted by the vector $\mathbf{s}_{j}^{*}$ and their $Z$-transform can be represented as $\mathbf{S}^{*}(z)=\sum_{j=0}^{M-1} z^{-i} \mathbf{S}_{j}^{*}\left(z^{M}\right)$. The length of $\mathbf{s}_{j}^{*}$ is $N_{s}=N / M$. Using the orthogonal analysis filters $\mathbf{h}_{0}, \cdots, \mathbf{h}_{M-1}$, the input signal, $x(n)$, and the desired signal, $d(n)$, are partitioned into new sets of subband signals $x_{i j}(n)$ and $d_{i}(n)$, respectively. The tap-update equation of SAP is given by

$$
\mathbf{S}(n+1)=\mathbf{S}(n)+\mu \mathbf{U}(n) \widetilde{\boldsymbol{\Phi}}^{-1}(n) \mathbf{E}(n) .
$$

In the above equation, $\mu$ is the step size, $\mathbf{S}(n)=\left[\mathbf{s}_{0}^{T}(n), \cdots, \mathbf{s}_{M-1}^{T}(n)\right]^{T}$ is the estimate of the polyphase tap vector of the $\mathrm{AF}$ at time instant $n . \mathbf{s}_{j}(n)$ is the $j$ th polyphase component of $\mathbf{S}(n)$ which is $N_{s} \times 1$ column vector. $\mathbf{U}(n)$ is an $M N_{s} \times M P_{s}$ input signal matrix [2, Eq. (38)], $\widetilde{\mathbf{\Phi}}(n)=\mathbf{\Phi}(n)+\delta \mathbf{I}_{p}$, where $\mathbf{I}_{p}$ is a $P \times P$ identity matrix and $\delta$ is a small regularization constant. $\boldsymbol{\Phi}(n)=\mathbf{U}^{T}(n) \mathbf{U}(n)=\operatorname{diag}\left(\mathbf{A}_{0}(n), \cdots, \mathbf{A}_{M-1}(n)\right)$ is dependent on the power of the input signal [2, Eq. (39)] and $\mathbf{A}_{i}(n)$ is a $P_{s} \times P_{s}$ matrix, diag denotes the diagonal elements. $\mathbf{E}(n)$ is an error vector which comprises of entries of the subband error signal $\mathbf{e}_{i}(n)$ [2, Eq. (40)].

Define the weight-error vector $\widetilde{\mathbf{S}}(n)=\left[\widetilde{\mathbf{s}}_{0}^{T}(n), \cdots, \widetilde{\mathbf{s}}_{M-1}^{T}(n)\right]^{T}$, where $\widetilde{\mathbf{s}}_{j}(n)=\mathbf{s}^{*}-\mathbf{s}_{j}(n)$. The disturbed error vector is defined as $\boldsymbol{\xi}(n)=\mathbf{U}^{T}(n) \widetilde{\mathbf{S}}(n)$. Assuming that $v_{i}(n)$ denotes the $i$ th subband component of the background noise $v(n)$ and its variance is $\sigma_{i, v}^{2}$. We obtain

$$
\mathbf{E}(n)=\boldsymbol{\xi}(n)+\mathbf{v}(n)
$$

where $\mathbf{v}(n)=\left[\mathbf{v}_{0}^{T}(n), \cdots, \mathbf{v}_{(M-1)}^{T}(n)\right]^{T}$ and $\mathbf{v}_{i}(n)=\left[v_{i}(n), \cdots, v_{i}\left(n-P_{s}+\right.\right.$ $1)]^{T}$. The mean square deviation (MSD) is defined as $D(n)=E\left\{\widetilde{\mathbf{S}}^{T}(n) \widetilde{\mathbf{S}}(n)\right\}$.

We use the optimal step size, $\mu_{\mathrm{opt}}(n)$, in place of the fixed step size in (1) and the DC-VSS-SAP is established. $\mu_{\mathrm{opt}}(n)$ is given by [3]

$$
\mu_{\mathrm{opt}}(n)=\frac{\widehat{D}(n) \cdot \operatorname{tr}\left(\boldsymbol{\Phi}(n) \widetilde{\boldsymbol{\Phi}}^{-1}(n)\right)}{N \cdot \operatorname{tr}\left(R_{E}(n) \widetilde{\boldsymbol{\Phi}}^{-1}(n)\right)}
$$

where $R_{E}(n)$ is computed as $R_{E}(n)=\alpha R_{E}(n-1)+(1-\alpha) \mathbf{E}(n) \mathbf{E}^{T}(n)$ and $\alpha$ is a smoothing constant. tr denotes the trace of a matrix. $\widehat{D}(n)$ is the estimate of MSD calculated by the method of delay coefficients [3, Eq. (7)] which can be achieved by delaying the input by $b$ samples.

\section{VSS-SAP with dynamic selection of subband filters}

Considering the weight-error vector $\widetilde{\mathbf{S}}(n)$ and variable step size $\mu(n)$, we can get

$$
\widetilde{\mathbf{S}}(n+1)=\widetilde{\mathbf{S}}(n)-\mu(n) \mathbf{U}(n) \widetilde{\boldsymbol{\Phi}}^{-1}(n) \mathbf{E}(n) .
$$

Squaring both sides, taking expectations and assuming no regularization is used in order to facilitate derivation, i.e., $\delta=0$, we can derive the MSD 
that satisfies

$$
\begin{aligned}
D(n+1)= & D(n)+\mu^{2}(n) E\left\{\mathbf{E}^{T}(n) \boldsymbol{\Phi}^{-1}(n) \mathbf{E}(n)\right\} \\
& -2 \mu(n) E\left\{\widetilde{\mathbf{S}}^{T}(n) \mathbf{U}(n) \boldsymbol{\Phi}^{-1}(n) \mathbf{E}(n)\right\} \\
\triangleq & D(n)-\Delta
\end{aligned}
$$

where $\Delta$ is related to the input matrix. If we maximize $\Delta$, then the fastest convergence is obtained as the MSD undergoes the largest decrease from iteration $n$ to $n+1$. Assuming that the noise variances for all subbands are the same, $\Delta$ can be rewritten as

$$
\Delta=\mu(n)[2-\mu(n)] E\left\{\mathbf{E}^{T}(n) \boldsymbol{\Phi}^{-1}(n) \mathbf{E}(n)\right\}-2 \mu(n) \sigma_{i, v}^{2} \operatorname{Tr}\left\{E\left[\boldsymbol{\Phi}^{-1}(n)\right]\right\}
$$

where we assume that the noise signal $v_{i}(n)$ is independent and identically distributed (i.i.d) and statistically independent of the subband data $\mathbf{U}(n)$, and we ignore the dependency of $\widetilde{\mathbf{S}}(n)$ on past noise [5]. Since it is not feasible to compute the exact expected value, we use an instantaneous value in replace of it as follows:

$$
\widehat{\Delta}=\mu(n)[2-\mu(n)] \mathbf{E}^{T}(n) \boldsymbol{\Phi}^{-1}(n) \mathbf{E}(n)-2 \mu(n) \sigma_{i, v}^{2} \operatorname{Tr}\left\{\boldsymbol{\Phi}^{-1}(n)\right\} .
$$

Substituting [2, Eq. (39), Eq. (40)] into (7), we get

$$
\begin{aligned}
\widehat{\Delta} & =\sum_{i=0}^{M-1} \widehat{\Delta}_{i} \\
& =\mu(n)[2-\mu(n)] \sum_{i=0}^{M-1}\left\{\mathbf{e}_{i}^{T}(n) \mathbf{A}_{i}^{-1}(n) \mathbf{e}_{i}(n)-2 \sigma_{i, v}^{2} \operatorname{Tr}\left[\mathbf{A}_{i}^{-1}(n)\right] /[2-\mu(n)]\right\}
\end{aligned}
$$

where $\widehat{\Delta}_{i}=\mu(n)[2-\mu(n)]\left\{\mathbf{e}_{i}^{T}(n) \mathbf{A}_{i}^{-1}(n) \mathbf{e}_{i}(n)-2 \sigma_{i, v}^{2} \operatorname{Tr}\left[\mathbf{A}_{i}^{-1}(n)\right] /[2-\mu(n)]\right\}$ is the contribution of the $i$ th subband input signal matrices, $\mathbf{X}_{i j}(n)(j \in$ $[0, \cdots, M-1]),\left[2\right.$, Eq. (12)] to the MSD. If $\widehat{\Delta}_{i}>0$, then it contributes to maximizing $\widehat{\Delta}$. However, if $\widehat{\Delta}_{i} \leq 0$, then it makes $\widehat{\Delta}$ decrease.

For colored inputs, $\boldsymbol{\Phi}(n)$ is a block diagonal matrix, and for white inputs, it is a diagonal matrix [3]. Thus, the matrix $\mathbf{A}_{i}(n)=\sum_{j=0}^{M-1} \mathbf{X}_{i j}^{T}(n) \mathbf{X}_{i j}(n)$ is a diagonal matrix for white inputs. Furthermore, for colored inputs, we assume that the diagonal components of $\mathbf{A}_{i}(n)$ are much larger than the off-diagonal components, we can focus only on the diagonal components of $\mathbf{A}_{i}(n)$. This solution is similar to the algorithm in [6]. Although the solution obtained from this assumption is not exactly same to the full implementation, it can decrease the computational complexity and retain satisfactory convergence performance. Hence, we can approximate $\widehat{\Delta}_{i}$ as

$$
\widehat{\Delta}_{i} \approx \mu(n)[2-\mu(n)] \sum_{k=0}^{P_{s}-1}\left\{\frac{e_{i}^{2}(n-k)-2 \sigma_{i, v}^{2} /[2-\mu(n)]}{a_{i, k}}\right\}
$$

where $a_{i, k}=\sum_{j=0}^{M-1} \mathbf{x}_{i j}^{T}(n-k) \mathbf{x}_{i j}(n-k)$ is the $k$ th diagonal component of $\mathbf{A}_{i}(n), \mathbf{x}_{i j}(n-k)$ is the $k$ th column vector of $\mathbf{X}_{i j}(n)[2$, Eq. (12)], $k \in$ $\left[0, \cdots, P_{s}-1\right]$.

From (9), we can calculate $\widehat{\Delta}_{i}$ and carry out the update with the $i$ th subband input matrices, $\mathbf{X}_{i j}(n)(j \in[0, \cdots, M-1])$, satisfying $\widehat{\Delta}_{i}>0$ for the largest MSD decrease at every iteration. Note that $a_{i, k}$ is dependent on 
the $i$ th subband input signal power and its fluctuation from one iteration to the next can be assumed to be small for a high-order adaptive filter [7], so we can only consider the numerator on the right side of (9) and approximate $\widehat{\Delta}_{i}>0$ as $\left\|\mathbf{e}_{i}(n)\right\|^{2}>2 P_{s} \sigma_{i, v}^{2} /[2-\mu(n)]$ for lower computational complexity, where $\|\cdot\|$ is the Euclidean norm of a vector.

Let $\Theta_{M(n)}=\left\{t_{1}, t_{2}, \cdots, t_{M(n)}\right\}$ denote a subset with $M(n)$ numbers of the set $\{0,1, \cdots, M-1\}$, where $t_{l}$ means the index of the selected subband filters, and $M(n)$ is defined as the number of the selected subband filters at iteration $n$. Then, the tap-update equation of the proposed algorithm becomes

$$
\mathbf{S}(n+1)=\left\{\begin{array}{lr}
\mathbf{S}(n), & \text { if } M(n)=0 \\
\mathbf{S}(n+1)=\mathbf{S}(n)+\mu(n) \mathbf{U}_{\Theta_{M(n)}}(n) & \\
\times \widetilde{\boldsymbol{\Phi}}_{\Theta_{M(n)}^{-1}}^{-1}(n) \mathbf{E}_{\Theta_{M(n)}}(n), & \text { otherwise }
\end{array}\right.
$$

where

$$
\begin{gathered}
\mathbf{U}_{\Theta_{M(n)}}(n)=\left[\begin{array}{cccc}
\mathbf{X}_{t_{1}, 0}(n) & \mathbf{X}_{t_{2}, 0}(n) & \cdots & \mathbf{X}_{t_{M(n)}, 0}(n) \\
\mathbf{X}_{t_{1}, 1}(n) & \mathbf{X}_{t_{2}, 1}(n) & \cdots & \mathbf{X}_{t_{M(n)}, 1}(n) \\
\vdots & \vdots & \ddots & \vdots \\
\mathbf{X}_{t_{1}, M-1}(n) & \mathbf{X}_{t_{2}, M-1}(n) & \cdots & \mathbf{X}_{t_{M(n)}, M-1}(n)
\end{array}\right], \\
\mathbf{E}_{\Theta_{M(n)}}(n)=\left[\begin{array}{c}
\mathbf{e}_{t_{1}}(n) \\
\mathbf{e}_{t_{2}}(n) \\
\vdots \\
\mathbf{e}_{t_{M(n)}}(n)
\end{array}\right]
\end{gathered}
$$

$\left\|\mathbf{e}_{t_{l}}(n)\right\|>\sqrt{2 P_{s} /[2-\mu(n)]} \sigma_{t_{l}, v}(l=1,2, \cdots, M(n))$ and $1 \leq M(n) \leq M$. $\mathbf{X}_{t_{l}, j}$ is the $t_{l}$ th subband input signal matrix whose form is same to $[2$, Eq. (12)] and $\widetilde{\boldsymbol{\Phi}}_{\Theta_{M(n)}}(n)=\mathbf{U}_{\Theta_{M(n)}^{T}}^{T}(n) \mathbf{U}_{\Theta_{M(n)}}(n)+\delta \mathbf{I}$. Furthermore, Substituting (3) into (10), the DS-VSS-SAP algorithm is established.

\section{Results and discussions}

To evaluate the performance of the proposed DS-VSS-SAP algorithm, we consider the system identification model of the echo cancellation problem. The input signals are zero-mean, wide-sense stationary AR(1) process which is given by $G(z)=1 /\left(1-0.9 z^{-1}\right)$. The cosine-modulated filter banks [1] with subband number $M=8$ are used and the length of their prototype filter, $L$, is set to 128 . When the fullband sampling rate is $F_{s}=1 / T_{s}$, the sampling rate of the subband structure is at a lower rate, $1 / M T_{s}$. The default values $P=16, S N R=30 \mathrm{~dB}$ and $\alpha=0.9$ are employed. The other parameters are $N=1024$ and $b=120$. The normalized MSD (NMSD), defined as $10 \log _{10} E\left[\widetilde{\mathbf{S}}^{T}(n) \widetilde{\mathbf{S}}(n)\right] /\left(\mathbf{s}^{* T} \mathbf{s}^{*}\right)$, is used as a performance measure. Also, we can assume that the subband noise variance, $\sigma_{i, v}^{2}$, is known, because it can be estimated efficiently [7].

Fig. 1 shows the NMSD curves for the SAP, DC-VSS-SAP and DS-VSSSAP by averaging over 100 independent trials. From [1], we know that the 
DS-VSS-SAP is stable if the step size satisfies $0<\mu<2$ regardless of the number of subbands. The step sizes are set to 1.2, 0.4, 0.1 for the SAP and the initial step sizes, $\mu(0)$, are also set to 1.2, 0.4, 0.1 for the DC-VSSSAP and DS-VSS-SAP. We can see that the DS-VSS-SAP obtains a lower steady-state NMSD compared to the other two algorithms.

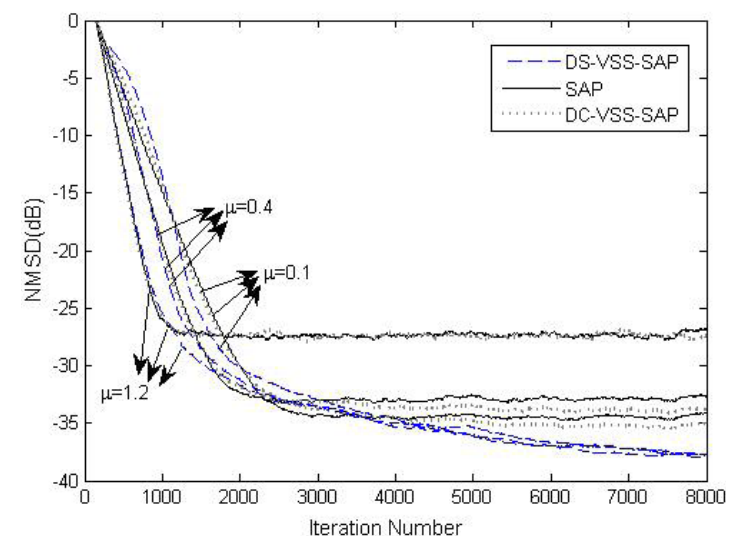

Fig. 1. NMSD curves of SAP, DC-VSS-SAP, DS-SAP and DS-VSS-SAP with $(M, P)=(8,16)$, AR $(1)$ input signal.

Fig. 2 (a) shows the number of selected subbands over 1 trial for the proposed DS-VSS-SAP with $M=8$ and the initial step size, $\mu(0)=1.2$. The average number of selected subbands over 100 independent trials is presented in. Fig. 2 (b). As can be seen, the selected subbands filters become small in the steady state.
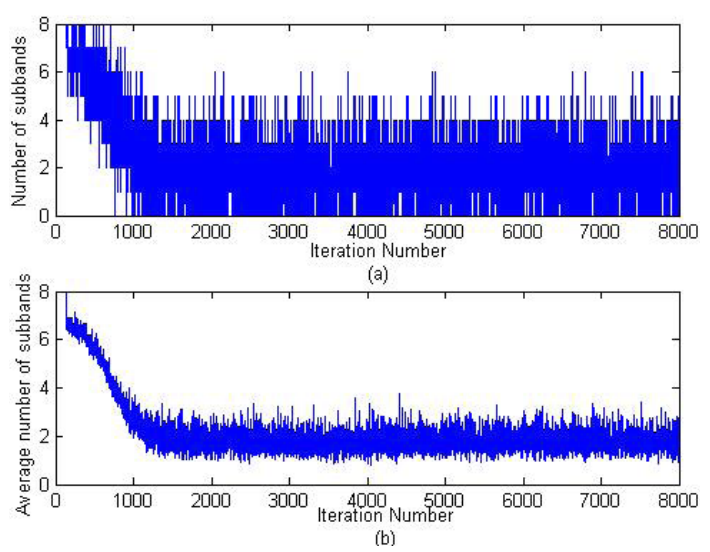

Fig. 2. Number of selected subbands for the DS-VSSSAP. (a) Number of selected subbands over single trial, and (b) Average number of selected subbands over 100 independent trials.

Table I shows the comparison of the computational complexities per it- 
obtained by averaging over the 500 steady-state samples from 100 trials with $\mu=1.2$ for the SAP and $\mu(0)=1.2$ for the DC-VSS-SAP and DS-VSS-SAP. For all three algorithms, the subband partitioning needs $2 M L$ multiplications. For error estimation, due to the increased $b$ taps of the adaptive filter for variable step size estimation, the DC-VSS-SAP and DS-VSS-SAP require $(N+b) P / M$ multiplications compared with $N P / M$ multiplications needed in the SAP. For tap-weight adaptation, the SAP needs $P^{3} /\left(2 M^{3}\right)+N P^{2}(M+$ 1) $/ M^{3}+N P(P+1) / M^{2}$ multiplications [2], while the DC-VSS-SAP and DSVSS-SAP require $P^{3} /\left(2 M^{3}\right)+(N+b) P^{2}(M+1) / M^{3}+(N+b) P(P+1) / M^{2}$ and $r(n)\left[P^{3} /\left(2 M^{3}\right)\right]+(N+b) P^{2} r(n)[r(n) M+1] / M^{3}+(N+b) P[r(n) P+1] / M^{2}$ multiplications respectively, where $r(n)=M(n) / M$. In addition, $\widetilde{\boldsymbol{\Phi}}(n)$ can be approximated to be a diagonal matrix [3], the calculation of variable step size for the DC-VSS-SAP and DS-VSS-SAP requires extra $3 P^{2}+2 P+b+2$ multiplications. Therefore, the DC-VSS-SAP employs $b P^{2}(M+1) / M^{3}+$ $b P(P+M+1) / M^{2}+3 P^{2}+2 P+b+2$ additional multiplications than the SAP due to the increased $b$ taps of the adaptive filter. For the DS-VSS-SAP, because of the small number of selected subband filters, the number of multiplication is smaller than that of the DC-VSS-SAP. On the other hand, the larger value of $b$ results in the increasing multiplications, as can be seen in Table I. Hence, the proposed DS-VSS-SAP has lower overall computational complexity compared to the SAP if and only if the following inequality holds

$$
b<\frac{[1-r(n)]\left\{P^{3} / 2+N P^{2}[r(n) M+M+1]+M N P^{2}\right\}-3 M^{3} P^{2}-2 M^{3} P-2 M^{3}}{M^{3}+r(n) P^{2}[r(n) M+1]+M P[r(n) P+M+1]} .
$$

Table I. Comparison of the computational complexities.

\begin{tabular}{|c|c|c|c|}
\hline Algorithm & Multiplications/iteration & $\begin{array}{l}L=128 \quad N=1024 \\
b=120 \quad, \quad M=8 \\
P=16 \quad \text { (simulated } \\
\text { multiplications) }\end{array}$ & $\begin{array}{l}L=128, N=1024 \\
b=1600 \quad, \quad M=8 \\
P=16 \quad \text { (simulated } \\
\text { multiplications) }\end{array}$ \\
\hline SAP & $\begin{array}{l}P^{3} /\left(2 M^{3}\right)+N P^{2}(M+1) / M^{3} \\
+N P(P+M+1) / M^{2}+2 M L\end{array}$ & $\approx 13088$ & $\approx 13088$ \\
\hline $\begin{array}{l}\text { DC-VSS- } \\
\text { SAP }\end{array}$ & $\begin{array}{l}P^{3} /\left(2 M^{3}\right)+(N+b) P^{2}(M+1) / M^{3} \\
+(N+b) P(P+M+1) / M^{2}+2 M L \\
+3 P^{2}+2 P+b+2\end{array}$ & $\approx 15300$ & $\approx 32690$ \\
\hline $\begin{array}{l}\text { DS-VSS- } \\
\text { SAP }\end{array}$ & $\begin{array}{l}r(n) P^{3} /\left(2 M^{3}\right)+(N+b) P^{2} r(n)[r(n) M+1] / M^{3} \\
+(N+b) P[r(n) P+M+1] / M^{2}+2 M L \\
+3 P^{2}+2 P+b+2\end{array}$ & $\approx 6938$ & $\approx 13541$ \\
\hline
\end{tabular}

\section{Conclusion}

A variable step size subband affine projection algorithm with dynamic selection of subband filters (DS-VSS-SAP) is proposed. It dynamically selects a subset of subband filters at every iteration based on maximizing the difference of successive MSDs. By dynamically selecting the significant subband filters, the proposed DS-VSS-SAP not only achieves a lower misadjustment compared with the existing SAP algorithms, but also lessens the computational burden. In addition, the simulation results show the efficiency of the proposed DS-VSS-SAP. 